$R M x A C, \mathbf{5 2}, 15-16(2020)$

(C) 2020: Instituto de Astronomía, Universidad Nacional Autónoma de México

https://doi.org/10.22201/ia.14052059p.2020.52.05

\title{
THE VARIABLE STARS OF CLEMENT CATALOGUE IN SOME SOUTHERN GLOBULAR CLUSTERS USING GAIA DR2
}

\author{
I. H. Bustos Fierro ${ }^{1}$ and J. H. Calderón ${ }^{1}$
}

RESUMEN

\begin{abstract}
En este trabajo se estudia la identificación y pertenencia a cúmulos globulares de las estrellas variables del Catalogue of Variable Stars in Galactic Globular Clusters de Christine Clement usando Gaia DR2. Se aborda el problema de la identificación cruzada. Se presentan resultados preliminares correspondientes a los cúmulos australes NGC 6362 y NGC 3201.
\end{abstract}

\begin{abstract}
In this work we study the identification and membership to globular clusters of stars in the Catalogue of Variable Stars in Galactic Globular Clusters by Christine Clement, using Gaia DR2. We discuss the issue of cross identification. We show preliminary results corresponding to the southern clusters NGC 6362 and NGC 3201.
\end{abstract}

Key Words: astrometry — stars: variables: general — globular cluster: general — globular clusters: individual: NGC 3201, NGC 6362

\section{INTRODUCTION}

Gaia DR2 (Gaia Collaboration 2018) -GDR2 hereafter- gives the most precise positions and proper motions with a limiting magnitude of $G \approx 21$ attainable up to the present. GDR2 also gives multicolor photometry making possible to perform colormagnitude diagrams (CMDs). These data allow to determine membership of stars to some stellar systems such as stellar clusters.

Most of the stars in the Catalogue of Variable Stars in Galactic Globular Clusters by Clement et al. (2001) and its updates - CCVS hereafter -, do not have astrometric membership determination. Different authors determine the membership of the variables by means of their positions in the CMDs of the cluster under study, and the membership of many variables has not been determined yet (see references in CCVS).

In this work - an application of the method described in "Membership determination in globular clusters with Gaia DR2 astrometry" by Bustos Fierro \& Calderón in these same proceedings - we do the cross identification of the stars in CCVS and GDR2, and determine their membership by means of positions and proper motions, which are later confirmed by their locations in the CMD constructed with Gaia photometry. We show the results for two southern globular clusters that have been observed

\footnotetext{
${ }^{1}$ Universidad Nacional de Córdoba. Observatorio Astronómico. Laprida 854, X5000BGR, Bo. Observatorio, Córdoba, Argentina (ivanbf@oac.unc.edu.ar).
}

by our team: NGC 3201 (Arellano Ferro et al. 2014) and NGC 6362 (Arellano Ferro et al. 2018).

\section{CROSS-MATCH}

In order to identify in GDR2 the stars in CCVS, we perform for each cluster a cross-match between the positions in the two catalogs using TopCat (Taylor 2005) with an identification radius of $2^{\prime \prime}$. Positions and mean magnitudes of the variable stars were taken from CCVS.

When performing the positional match three different situations arise: within $2^{\prime \prime}$ of the variable star there is no GDR2 source, there is only one GDR2 source, or there is more than one GDR2 source. This last situation appears in many variables.

Although the photometric systems in CCVS and in GDR2 are different, we have noticed that $\langle V\rangle$ in CCVS and $G_{m a g}$ in GDR2 are similar, therefore we use them as an additional criterion for the crossmatch. When there is more than one GDR2 source within $2^{\prime \prime}$ of the variable star, we consider them all as candidates to be the variable. Among those candidates we chose the one with the smallest difference in magnitudes $\left|G_{m a g}-\langle V\rangle\right|$ even if it is not the closest match. For example, in the case of V106 in NGC 3201 that has two candidates as shown in Figure 1, one at $1.1^{\prime \prime}$ with $\left|G_{m a g}-\langle V\rangle\right|=1.46 \mathrm{mag}$ and the other at $1.7^{\prime \prime}$ with $\left|G_{m a g}-\langle V\rangle\right|=0.03 \mathrm{mag}$, we chose the candidate located at $1.7^{\prime \prime}$. 
TABLE 1

SAMPLE OF THE TABLE OF VARIABLE STARS IN NGC 3201. EXPLANATION IN THE TEXT

\begin{tabular}{clllcccccl}
\hline Var & $\langle m a g\rangle$ & ampl & Type & Remarks & Memb. & Gaia-DR2 ID Source & Sep. $\left(^{\prime \prime}\right)$ & Id.Crit. & Notes \\
\hline V1 & 14.81 & 0.92 & RR0 & A & M & 5413575310452568064 & 0.08 & P & Astrometry confirms membership \\
V65 & 14.03 & 0.77 & E & max;Note;f? & F & 5413593491058124544 & 0.15 & P & Confirmed as field star. \\
V69 & 14.74 & 1.10 & RR0 & C84 & P & 5413588096579420800 & 0.15 & P & Possible member... \\
V104 & 17.46 & 0.02 & SXP & & M & 5413576517342565632 & 1.76 & P $+\Delta m$ & Dubious cross-match... \\
V113 & 11.89 & 0.02 & LB & Field? & M & 5413574833720518528 & 0.13 & P & Located on the GB in the CMD. \\
V119 & 16.049 & 0.20 & EA & max;Note & U & 5413575452190327424 & 0.07 & P & Proper motion not available. \\
V122 & 18.107 & 0.05 & SXP & f & M & 5413575555269322752 & 0.69 & P & Membership status disagrees... \\
V149 & 21.28 & 0.48 & EB? & & U & & & & Not found in Gaia-DR2. \\
\hline
\end{tabular}

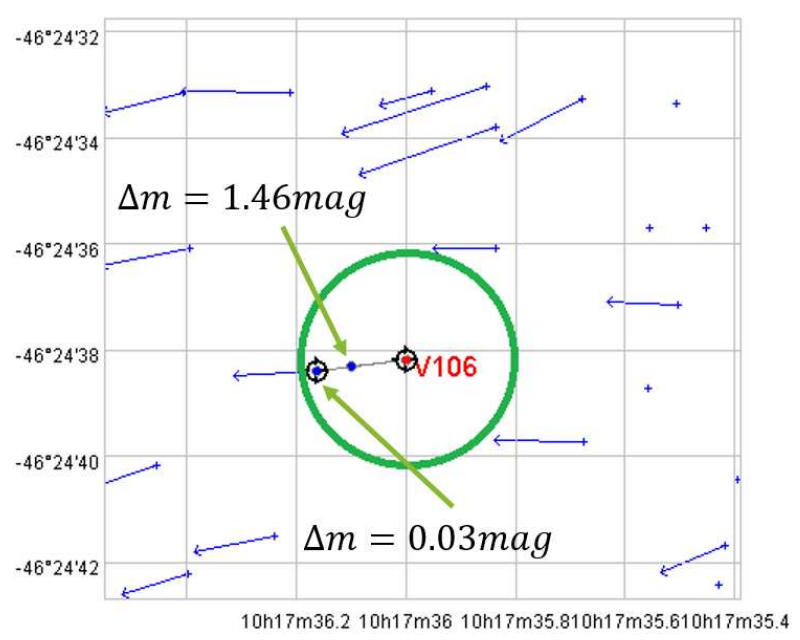

Fig. 1. CCVS-GAIA DR2 cross match. CCVS position of V106 in NGC 3201 (red dot), the 2" cross match radius (green circle) and and the two GDR2 sources with their proper motions (blue dots and arrows), labeled by their $\Delta m=\left|G_{m a g}-\langle V\rangle\right|$

\section{RESULTS}

Once the cross-match between CCVS and GDR2 was determined, we consider the variable star is a member of the cluster $(\mathrm{M})$, a possible member $(\mathrm{P})$, or a field star (F) as found for the corresponding GDR2 source, after application of the method described in the contribution by Bustos Fierro \& Caderón mentioned above. For the variables without match in GDR2 or a match without proper motion in GDR2, their memberships remain unknown (U).

Table 1 shows some lines of the table that summarises the results corresponding to the variables in NGC 3201: Columns 1-5 are from CCVS, Column 6 is the membership status as explained, Column 7 is the Gaia-DR2 ID Source that was cross-matched with the corresponding variable in CCVS, Column 8 is the separation between the position of the variable in CCVS and the source in GDR2, Column 9 indicates if the match criterion was only position $(\mathrm{P})$ or position and magnitude difference $(\mathrm{P}+\Delta m)$.

\section{FINAL REMARKS}

The method developed for the cross-match between the CCVS and GaiaDR2 makes use of positions and magnitudes, since positions only are not enough in the dense regions of globular clusters. Almost all the variables in the CCVS in the fields of NGC 3201 and NGC 6362 were successfully identified in GaiaDR2. The median separations between positions in CCVS and GaiaDR2 are similar in both clusters, around 1.6". This is the first astrometric GaiaDR2-based membership determination for most of the variables in these clusters. Some previously known members were confirmed, as well as some field stars. In some cases our membership status disagrees with previous determinations.

Acknowledgements: This research has made use of the software TOPCAT (Taylor 2005).

\section{REFERENCES}

Arellano Ferro A., Ahumada J. A., Bustos Fierro I. H., Calderón J. H. \& Morrell N. I. 2018, AN, 339, 183

Arellano Ferro A., Ahumada J. A., Calderón J. H., \& Kains N. 2014, RMxAA, 50, 307

Clement, C. M., Muzzin, A., Dufton, Q., et al. 2001, AJ, 122,2587

Gaia Collaboration et al. 2018, A\&A, 616, A1

Taylor, M. B. 2005, in Astronomical Data Analysis Software and Systems XIV, ed. P. Shopbell, M. Britton, \& R. Ebert, ASP Conf. Series, 347, 29 